VERsion August 20, 2019: FM

Preprint typeset using LATEX style emulateapj v. 11/10/09

\title{
UNVEILING THE NATURE OF THE UNIDENTIFIED GAMMA-RAY SOURCES V: ANALYSIS OF THE RADIO CANDIDATES WITH THE KERNEL DENSITY ESTIMATION
}

\author{
F. Massaro ${ }^{1}$, R. D'Abrusco ${ }^{2}$, A. PagGi $^{2}$, N. Masetti ${ }^{3}$, \\ M. Giroletti ${ }^{4}$, G. Tosti $^{5}$, Howard, A. Smith $^{2}$, \& S. Funk ${ }^{1}$. \\ version August 20, 2019: fm
}

\begin{abstract}
Nearly one-third of the $\gamma$-ray sources detected by Fermi are still unidentified, despite significant recent progress in this effort. On the other hand, all the $\gamma$-ray extragalactic sources associated in the second Fermi-LAT catalog have a radio counterpart. Motivated by this observational evidence we investigate all the radio sources of the major radio surveys that lie within the positional uncertainty region of the unidentified $\gamma$-ray sources (UGSs) at $95 \%$ level of confidence. First we search for their infrared counterparts in the all-sky survey performed by the Wide-field Infrared Survey Explorer $(W I S E)$ and then we analyze their IR colors in comparison with those of the known $\gamma$-ray blazars. We propose a new approach, based on a 2-dimensional kernel density estimation (KDE) technique in the single [3.4]-[4.6]-[12] $\mu \mathrm{m}$ WISE color-color plot, replacing the constraint imposed in our previous investigations on the detection at $22 \mu \mathrm{m}$ of each potential IR counterpart of the UGSs with associated radio emission. The main goal of this analysis is to find distant $\gamma$-ray blazar candidates that, being too faint at $22 \mu \mathrm{m}$, are not detected by WISE and thus are not selected by our purely IR based methods. We find fifty-five UGS's likely correspond to radio sources with blazar-like IR signatures. Additional eleven UGSs having, blazar-like IR colors, have been found within the sample of sources found with deep recent ATCA observations.

Subject headings: galaxies: active - galaxies: BL Lacertae objects - radiation mechanisms: non-thermal
\end{abstract}

\section{INTRODUCTION}

The large majority of the point sources detected by the Compton Gamma-ray Observatory in the 1990s (e.g., Hartman et al. 1999) are still lacking an association with a low-energy candidate counterpart, and given their sky distribution, a significant fraction of these unresolved objects are expected to have extragalactic origin (e.g., Thompson 2008; Abdo et al. 2010a). Unveiling the origin of the unidentified $\gamma$-ray sources (UGSs) is also one of the key scientific objectives of the recent Fermi mission that still lists about $1 / 3$ of the $\gamma$-ray sources as unassociated in the second Fermi-LAT catalog (2FGL; Nolan et al. 2012).

A large fraction of UGSs is expected to be blazars, the largest known population of $\gamma$-ray active galaxies, not yet associated and/or recognized due to the lack of multifrequency observations (Ackermann et al. 2011a). Therefore a better understanding of the nature of the UGSs is crucial to estimate accurately the blazar contribution to the extragalactic gamma-ray background (e.g., Mukherjee et al. 1997; (Abdo et al. 2010b), and it is essential to constrain exotic high-energy physics phenomena (e.g., Zechlin et al. 2012).

Many attempts have been adopted to decrease UGSs

\footnotetext{
${ }^{1}$ SLAC National Laboratory and Kavli Institute for Particle Astrophysics and Cosmology, 2575 Sand Hill Road, Menlo Park, CA 94025, USA

${ }^{2}$ Harvard - Smithsonian Astrophysical Observatory, 60 Garden Street, Cambridge, MA 02138, USA

${ }^{3}$ INAF - Istituto di Astrofisica Spaziale e Fisica Cosmica di Bologna, via Gobetti 101, 40129, Bologna, Italy

${ }^{4}$ INAF Istituto di Radioastronomia, via Gobetti 101, 40129, Bologna, Italy

${ }^{5}$ Dipartimento di Fisica, Università degli Studi di Perugia, 06123 Perugia, Italy
}

number and to understand their composition. Pointed Swift observations

(e.g., Mirabal 2009; $\quad$ Mirabal \& Halpern 2009; Paggi et al. 2013) to search for X-ray counterparts of UGSs as well as radio follow up observations were already performed or are still in progress (e.g., Kovalev 2009a; Kovalev et al. 2009b; Petrov et al. 2013). In addition, statistical approaches based on different techniques have been also developed and successfully used (e.g. Mirabal \& Pardo 2010; Ackermann et al. 2012).

We recently addressed the problem of searching $\gamma$ ray blazar candidates as counterparts of the UGSs adopting two new approaches: the first is based on the Wide-field Infrared Survey Explorer (WISE) allsky observations (Wright et al. 2010) aiming at recognizing $\gamma$-ray blazar candidates using their peculiar IR colors (Massaro et al. 2011a; D'Abrusco et al. 2012; Massaro et al. 2012b; D'Abrusco et al. 2013) while the second employs the low-frequency radio observations (Massaro et al. 2013b). In particular, this second method was indeed based on the combination of the radio observations Westerbork Northern Sky Survey (WENSS; Rengelink et al. 1997) at $325 \mathrm{MHz}$ with those of the NRAO Very Large Array Sky survey (NVSS; Condon et al. 1998) and of the Very Large Array Faint Images of the Radio Sky at Twenty-Centimeters (FIRST; Becker et al. 1995; White et al. 1997) at about $1.4 \mathrm{GHz}$.

It is worth noting that all the Fermi extragalactic sources associated in the 2FGL catalog have a clear radio counterpart (Nolan et al. 2012), this is the basis of the radio- $\gamma$-ray connection, that has been found in the case of blazars (e.g., Ghirlanda et al. 2010; Mahony et al. 2010; Ackermann et al. 2011b). Thus, motivated by this observational evidence we propose a different approach to search for the blazar-like counterparts of the UGSs. We 
combine the radio and the IR information available for the sources lying within the positional uncertainty regions of the Fermi UGSs to select $\gamma$-ray blazar candidates.

With respect to our previous IR based search for blazar-like counterparts

(e.g., Massaro et al. 2012a; D'Abrusco et al. 2013) our new analysis relaxes the constraint on the $22 \mu \mathrm{m}$ detection of the WISE-selected candidates, and does not take into account their [12]-[22] $\mu \mathrm{m}$ color, replacing these features with the presence of a radio counterpart. The number of $\gamma$-ray blazars undetected at $22 \mu \mathrm{m}$ is only a small fraction ( $\sim 8 \%$ of the total number of $\gamma$-ray blazars D'Abrusco et al. 2013), but includes several high redshift sources that lying at larger distance than the whole population.

To perform our analysis, we search all the radio sources detected in the

NVSS (Condon et al. 1998) and in the Sydney University Molonglo Sky Survey (SUMSS; Mauch et al. 2003) surveys that lie within the positional uncertainty region, at $95 \%$ level of confidence, of the UGSs listed in the 2FGL. Then we associate them with their WISE counterparts to compare their IR colors with those of the known $\gamma$-ray blazars in the [3.4]-[4.6]-[12] $\mu \mathrm{m}$ plot using the kernel density estimation (KDE) technique (e.g., Richards et al. 2004; D'Abrusco et al. 2009; Massaro et al. 2012a). We also verified if the radio sources found in the recent deep radio observations performed by Australia Telescope Compact Array (ATCA) and presented by Petrov et al. (2013) have an IR counterpart with WISE colors consistent with those of the $\gamma$-ray blazar population. Our analysis of the IR colors is restricted only to the [3.4]-[4.6]-[12] $\mu \mathrm{m}$ color-color plot.

The paper is organized as follows: Section 2 is devoted to the definitions of the samples used while in Section 3 we describe the KDE technique used to perform our investigation; we then applied our selection in Section 4 to identify those radio sources that could be considered blazar-like counterpart of the UGSs listed in the 2FGL catalog. We also verified the presence of optical and Xray counterparts for the selected $\gamma$-ray blazar candidates and we compare our results with different approaches previously developed. Finally, Section 5 is dedicated to our conclusions.

For our numerical results, we use cgs units unless stated otherwise. Spectral indices, $\alpha$, are defined by flux density, $\mathrm{S}_{\nu} \propto \nu^{-\alpha}$ and WISE magnitudes at the [3.4], [4.6], [12], [22] $\mu \mathrm{m}$ (i.e., the nominal WISE bands) are in the Vega system respectively. All the magnitudes and the IR colors reported in the paper have been corrected for the Galactic extinction according to the formulae reported in Draine (2003) as also performed in our previous analysis (e.g., D'Abrusco et al. 2013; Massaro et al. 2013a). The most frequent acronyms used in the paper are listed in Table 1

\section{SAMPLE SELECTION}

The first sample used in our analysis lists all the blazars listed in the Multiwavelength Blazar Catalog (ROMA-BZCAT, Massaro et al. 2009) that have been associated as counterparts of Fermi sources in the 2FGL

\footnotetext{
${ }^{6}$ http://www.asdc.asi.it/bzcat/
}

TABLE 1

LIST OF MOST FREQUENT ACRONYMS.

\begin{tabular}{|lc|}
\hline Name & Acronym \\
\hline Multifrequency Catalog of blazars & ROMA-BZCAT \\
Second Fermi Large Area Telescope Catalog & 2FGL \\
\hline BL Lac object & BZB \\
Flat Spectrum Radio Quasar & BZQ \\
Blazar of Uncertain type & BZU \\
Unidentified Gamma-ray Source & UGS \\
\hline Training Blazar sample & TB \\
Northern UGS sample & NU \\
Southern UGS sample & SU \\
Southern Deep ATCA sample & SDA \\
\hline Kernel Density Estimation & KDE \\
\hline
\end{tabular}

(Nolan et al. 2012) with a WISE counterpart detected at least in the first three filters regardless of the fact that they are detected at $22 \mu \mathrm{m}$. The association radius between the ROMA-BZCAT catalog and the WISE all-sky survey adopted here was fixed to $3^{\prime \prime} .3$ (see D'Abrusco et al. 2013, for more details). This sample, named training blazar (TB) sample, comprises a total of 737 blazars, excluding those classified as blazars of uncertain type (BZUs) (see also Massaro et al. 2010; Massaro et al. 2011b). The TB sample is used to build the isodensity contours for the KDE technique (see following sections) and to test if IR sources with radio counterparts have WISE colors consistent with the $\gamma$-ray blazar population.

Then the UGSs sample considered is the one constituted by all the Fermi sources listed in the 2FGL with no assigned counterpart at low energies and without any $\gamma$ ray analysis flag listing 299 sources (Nolan et al. 2012). We further divided this sample in two subsamples: the northern UGS (NU) sample where only sources with Declination above than $-40 \mathrm{deg}$ and the southern UGS (SU) sample selecting those at Declination below $-30 \mathrm{deg}$. This subdivision has been chosen on the basis of the footprints of the radio surveys used for our analysis, since the NU sample is mainly covered by the NVSS survey (Condon et al. 1998), while the SU one by the SUMSS catalog (Mauch et al. 2003). The former sample lists 209 UGSs while 115 sources belong to the latter one.

Finally, we also considered the list of all the radio sources recently found by Petrov et al. (2013) using deep ATCA observations for the UGSs in the southern hemisphere. This sample is labeled as southern deep ATCA (SDA) sample.

\section{KERNEL DENSITY ESTIMATION}

The KDE technique is a non-parametric procedure to estimate the probability density function of a multivariate distribution without requiring any assumption about the shape of the "parent" distribution. The KDE technique also permits to reconstruct the density distribution of a population of points in a general Ndimensional space based on a finite sample. This analysis depends on only one parameter, the bandwidth of the kernel of the density estimator (analogous to the window size for one-dimensional running average) that can be estimated locally (see e.g., Richards et al. 2004; D'Abrusco et al. 2009; Laurino \& D'Abrusco 2011, and reference therein). 
We already applied the KDE technique in several cases to compare the IR colors of blazar candidates selected with different procedures with those of the known population of $\gamma$-ray blazars (see Massaro et al. 2011a; Massaro et al. 2012a; Paggi et al. 2013, for more details). Thus in the present analysis we use the KDE method to compare the IR colors of the radio selected counterparts with those of the $\gamma$-ray blazar population represented by the TB sample in the 2-dimensional [3.4][4.6]-[12] $\mu \mathrm{m}$ color-color plot. As already described in Massaro et al. (2012a), we provide an associated confidence $\pi_{k d e}$ drawn from the KDE density probabilities that a selected radio source as IR colors consistent with the blazars in the TB sample.

In Figure 1 we show the density profiles constructed for the whole blazar population (left panel) and used to estimate $\pi_{k d e}$ and those of the two subsamples of BZBs and BZQs (right panel) belonging to the TB sample, to highlight the dichotomy between the two subclasses.

\section{UNIDENTIFIED $\gamma$-RAY SOURCES}

\subsection{Selection of $\gamma$-ray blazar candidates}

For each UGS we searched for all the radio sources that lie within their positional uncertainty regions at $95 \%$ level of confidence and we found that there are 822 radio sources potential counterparts of 209 UGSs and 134 out of 115 for the NU and the SU samples, respectively. We then crossmatched all these radio sources with the WISE all-sky catalog7 (Wright et al. 2010) using the same radius of $3^{\prime \prime} .3$ and we selected only those with an IR counterpart detected at least in the first three WISE filters and not extended (i.e., extension flag, ext_flg $\leq 1$ ) (Cutri et al. 2012). The $3^{\prime \prime} .3$ radius chosen to associated sources between the WISE and the radio catalogs is statistically justified on the basis of the analysis performed over the entire ROMA-BZCAT (see D'Abrusco et al. 2013, for more details). Thus we obtained 374 out of 822 and 78 out of 134 radio sources in the NU and SU samples, respectively.

Subsequently, we applied the KDE technique described in Section 3 to find radio sources with WISE counterparts having IR colors consistent with the $\gamma$-ray blazar population. We considered reliable $\gamma$-ray blazar candidates only radio sources consistent within the isodensity contours, drawn from the KDE, at $90 \%$ level of confidence, correspondent to an association confidence $\left(\pi_{k d e}\right)$ grater than 10.0 .

We found 41 and 14 radio sources WISE selected with $\pi_{k d e}>0.1$ within the NU and the SU samples, respectively. In addition, only 11 out of 416 radio sources listed in the SDA sample have an IR counterpart consistent with the Fermi blazar population of the TB sample with $\pi_{k d e}>10.0$. We also list two exceptions to the above criteria: the UGS 2FGLJ1223.3+7954 with its WISE blazar candidate WISE J122358.17+795327.8 in the NU sample and 2FGLJ0523.3-2530 with WISE J052313.07-253154.4 as potential counterpart in the SDA sample, having the $\pi_{k d e}$ values equal to 9.6 and 9.5 , respectively, marginally below our threshold. The total number of $\gamma$-ray blazar candidates is 66 all listed in Table 2 and Table 3 . It is worth noting that we do not have any multiple $\gamma$ -

7 http://wise2.ipac.caltech.edu/docs/release/allsky/ ray blazar candidate within the positional uncertainty regions of the UGSs analyzed.

In Figure 2 we show the isodensity contours derived from the KDE analysis in the [3.4]-[4.6]-[12] $\mu \mathrm{m}$ color color plot, together with the $\gamma$-ray blazar candidates selected in the UGS samples analyzed and in the SDA list. It is evident how the large fraction for the selected candidates are located within with the isodensity contours drawn for the BZB class.

To establish if the $\gamma$-ray blazar candidate selected with our method have additional multifrequency properties that could confirm their nature and provide redshift estimates, we also searched for the counterpart of our radio-IR selected candidates in the following major surveys. For the near-IR we used only the Two Micron All Sky Survey (2MASS; Skrutskie et al. 2006, - M) since each WISE source is already associated with the closest 2MASS source by the default cata$\log$ (see Cutri et al. 2012, for more details). We then searched for optical counterparts, with possible spectra available, in the Sloan Digital Sky Survey (SDSS; e.g. Adelman-McCarthy et al. 2008; Paris et al. 2012, - s), in the Six-degree-Field Galaxy Redshift Survey (6dFGS; Jones et al. 2004; Jones et al. 2009, - 6), in the The Muenster Red Sky Survey (MRSS; Ungruhe et al. 2003) and in the USNO-B Catalog (Monet et al. 2003) within $3^{\prime \prime} .3$. These optical cross correlations are also useful to plan follow up observations thus a complete list of sources together with their optical magnitudes is reported in Table 4. For the high energy we looked in the soft Xrays using the ROSAT all-sky survey catalog (RASS; Voges et al. 1999, - X). Finally, we also considered the NASA Extragalactic Database (NED) 8 for any possible counterpart within $3^{\prime \prime} .3$ for additional information. The results of this multifrequency investigation is presented and summarized in Table 2 and Table 3.

\subsection{Probability of spurious associations}

We estimated the probability that our $\gamma$-ray blazar candidates can be spurious associations adopting the following approach, similar to that successfully used in our previous analyses (e.g., Massaro et al. 2013b; Paggi et al. 2013).

We created two fake $\gamma$-ray catalogs shifting the coordinates of the $41 \gamma$-ray blazars in the NU sample and of the 25 in the $\mathrm{SU}$ one by $0^{\circ} .7$ in a random direction of the sky within the footprints of the NVSS and the SUMSS radio surveys. Keeping the same values of $\theta_{95}$ of each fake UGS, we verified that there were no correspondences with real Fermi sources within a circular region of radius $\theta_{95}$ at the flux level of the $2 \mathrm{FGL}$.

For each fake UGSs, we search for all the radio sources lying within the positional uncertainty region at $95 \%$ of confidence in both the NVSS and SUMSS radio surveys. We then checked the presence of an IR counterpart of each radio source selected above crossmatching the WISE all-sky catalog with their NVSS and SUMSS positions within a radius of $3^{\prime \prime} .3$. The value of this IRto-radio association radius has been chosen on the basis of our previous statistical analyses (see Section 2 and D'Abrusco et al. 2013, for more details).

8 http://ned.ipac.caltech.edu/ 

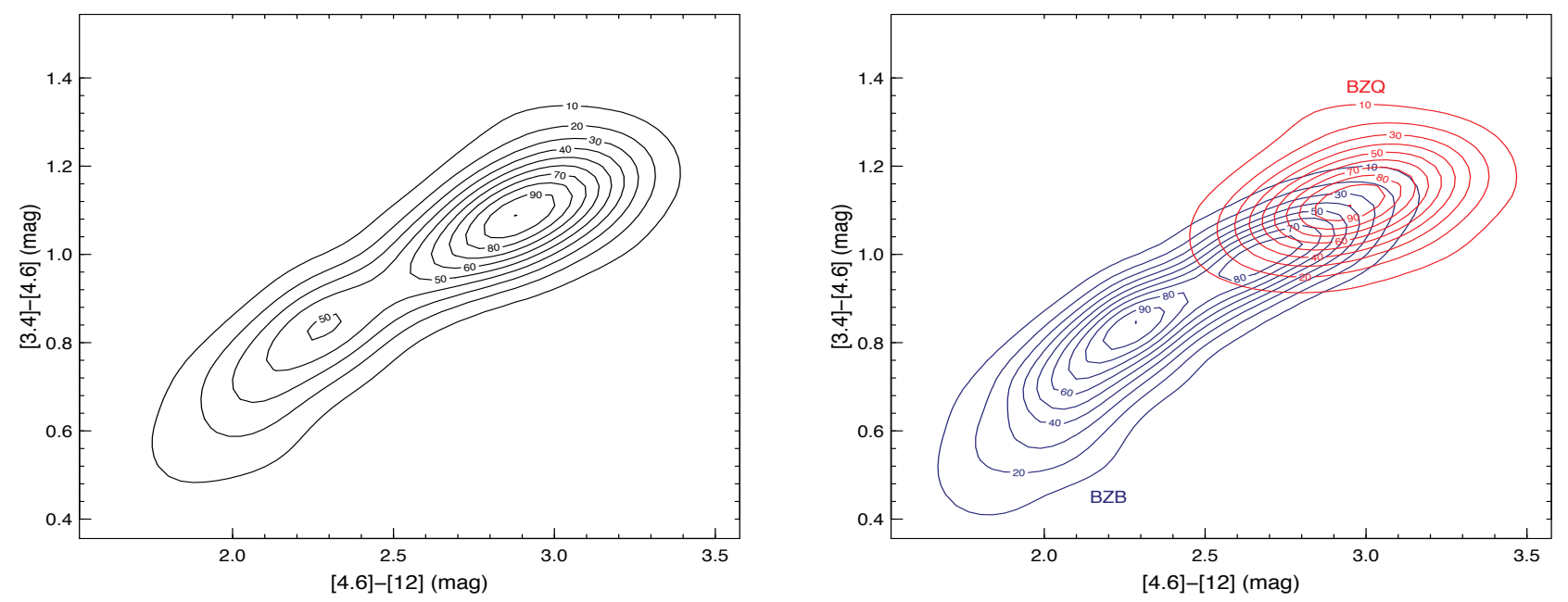

FIG. 1.- Left) The isodensity contours generated by KDE technique in the [3.4]-[4.6]-[12] $\mu \mathrm{m}$ color-color diagram for the whole $\gamma$-ray blazar population represented by the sources in the TB sample. Right) The KDE isodensity contours built separately for the BZB (blue) and the BZQ (red) classes in the TB sample. The numbers appearing close to each contour corresponds to the values of $\pi_{k d e}$ in both panels.

For each radio source with a WISE counterpart we applied our KDE technique selecting the radio sources detected by WISE at $3.4 \mu \mathrm{m}, 4.5 \mu \mathrm{m}$ and $12 \mu \mathrm{m}$ with $\pi_{k d e}>0.10$ being fake $\gamma$-ray blazar candidates. Then we repeated the entire procedure 10 times for both the $\mathrm{NU}$ and the SU sample to establish the probability of spurious associations. Based on the above procedure, we expect that $4 \%$ and $3 \%$ of the $\gamma$-ray blazar candidates previously selected for the UGS in the NU and SU samples respectively, could be contaminants.

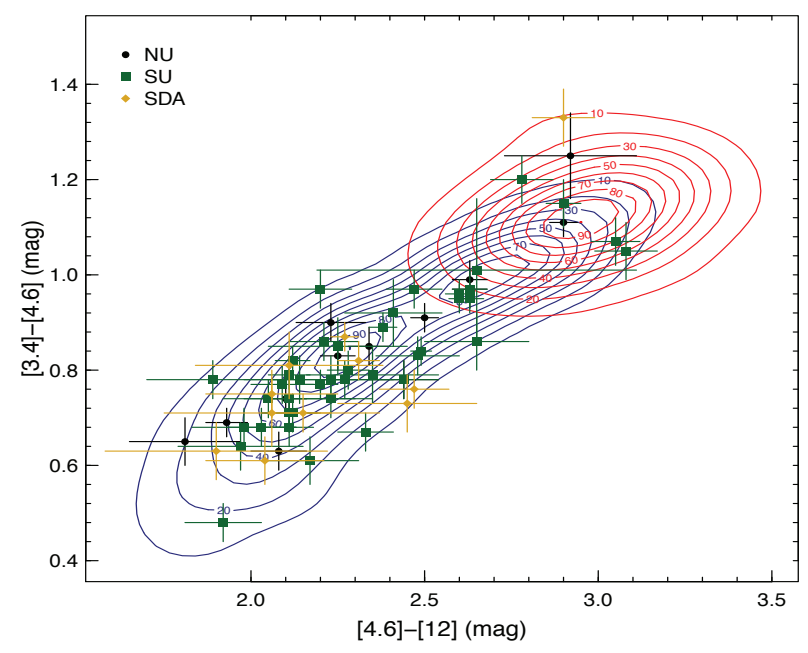

FIG. 2.- The isodensity contours generated by KDE technique in the [3.4]-[4.6]-[12] $\mu \mathrm{m}$ color-color diagram for the BZBs (blue) and the BZQs (red) in the TB sample. Points overlaid to the contours show the location of the selected radio candidates with IR colors consistent with the $\gamma$-ray blazar population within $\pi_{k d e}>10$ for the sources in the three different samples analyzed: NU (black circles), SU (green squares) and SDA (yellow diamonds). The numbers appearing close to each contour corresponds to the values of $\pi_{k d e}$.
Finally, we emphasize that these estimates depend on the $\gamma$-ray background model, the detection threshold and the flux limit of the 2FGL catalog (Nolan et al. 2012), in which no $\gamma$-ray emission is arising from any of the positions listed in the fake $\gamma$-ray catalogs.

\subsection{Comparison with previous investigations}

We compare our results with those of previous analyses carried out in Massaro et al. (2013a), Massaro et al. (2013b) and Paggi et al. (2013). The results of our comparison is summarized below and presented in Table 2 and Table 3 .

We note that within the $41 \gamma$-ray blazar candidates found in the NU sample there are 16 sources that were also selected on the basis of their three WISE colors in Massaro et al. (2013a) 7 that appeared as potential counterpart in Massaro et al. (2013b) found with the lowfrequency radio observations and 14 listed with an X-ray properties in Paggi et al. (2013). In addition, 12 UGS were also investigated in our previous analyses but for them we found a different $\gamma$-ray blazar candidate. The number of new candidates counterparts in the NU sample is 5. On the other hand, within the SU sample, we found that 8 radio sources were also selected in Massaro et al. (2013a) and 4 in Paggi et al. (2013), in addition to 4 new $\gamma$-ray blazar candidates.

Petrov et al. (2013) already found the WISE counterparts of their SDA sample but they did not verified which have IR colors consistent with the Fermi blazars. Thus in the SDA sample we listed 11 radio sources detected thanks to the deeper radio survey performed with ATCA (Petrov et al. 2013) with IR colors consistent with those of the $\gamma$-ray blazar population. Among these $11 \gamma$-ray blazar candidates, there are two sources already found in Massaro et al. (2013a) and only one UGS (i.e., 2FGLJ0547.5-0141c) previously investigated that appear to have a different potential counterpart.

We note that the comparison between the $\gamma$-ray blazar candidates found in the $\mathrm{SU}$ and in the SDA samples 
TABLE 2

Unidentified Gamma-ray Sources in the Northern and in the Southern samples.

\begin{tabular}{|c|c|c|c|c|c|c|c|c|}
\hline $\begin{array}{l}2 \mathrm{FGL} \\
\text { name }\end{array}$ & $\begin{array}{l}\text { WISE } \\
\text { name }\end{array}$ & $\begin{array}{l}\text { Radio } \\
\text { name }\end{array}$ & $\begin{array}{c}{[3.4]-[4.6]} \\
\mathrm{mag}\end{array}$ & $\begin{array}{c}{[4.6]-[12]} \\
\mathrm{mag}\end{array}$ & $\pi_{k d e}$ & notes & $\mathrm{z}$ & compare \\
\hline \multicolumn{9}{|c|}{ NORTHERN UGS SAMPLE } \\
\hline 2 FGLJ0031.0+0724 & J003119.70+072453.6 & NVSSJ003119+072456 & $0.83(0.04)$ & $2.48(0.12)$ & 29.3 & $\mathrm{~N}$ & $?$ & 3 \\
\hline 2FGLJ0039.1+4331 & J003908.14+433014.6 & NVSSJ003907+433015 & $0.97(0.04)$ & $2.20(0.09)$ & 10.3 & $\mathrm{~N}, \mathrm{v}$ & ? & $1,2,3$ \\
\hline 2FGLJ0103.8+1324 & J010345.73+132345.4 & NVSSJ010345+132346 & $0.68(0.04)$ & $2.03(0.10)$ & 31.3 & $\mathrm{~N}, \mathrm{M}$ & ? & 3 \\
\hline 2FGLJ0158.4+0107 & J015852.76+010132.9 & NVSSJ015852+010133 & $0.85(0.06)$ & $2.25(0.20)$ & 49.1 & $\mathrm{~N}, \mathrm{~F}, \mathrm{~s}, \mathrm{rv}$ & ? & - \\
\hline 2FGLJ0158.6+8558 & J015248.80+855703.6 & NVSSJ015248+855706 & $1.07(0.05)$ & $3.05(0.07)$ & 65.6 & $\mathrm{~N}, \mathrm{M}$ & ? & 1,2 \\
\hline 2 FGLJ0227.7+2249 & J022744.35+224834.3 & NVSSJ022744+224834 & $0.95(0.03)$ & $2.60(0.03)$ & 53.1 & $\mathrm{~N}, \mathrm{v}$ & ? & $1 !, 3 !$ \\
\hline 2FGLJ0312.8+2013 & J031240.54+201142.8 & NVSSJ031240+201141 & $0.79(0.06)$ & $2.35(0.19)$ & 36.4 & $\mathrm{~N}$ & ? & - \\
\hline 2FGLJ0332.1+6309 & J033153.90+630814.1 & NVSSJ033153+630814 & $0.96(0.03)$ & $2.60(0.04)$ & 54.5 & $\mathrm{~N}, \mathrm{M}$ & ? & $1 !, 2 !$ \\
\hline 2FGLJ0353.2+5653 & J035309.54+565430.8 & NVSSJ035309+565431 & $0.78(0.04)$ & $1.89(0.19)$ & 10.9 & $\mathrm{~N}, \mathrm{M}, \mathrm{rv}$ & ? & $2 !, 3 !$ \\
\hline 2FGLJ0409.8-0357 & J040946.57-040003.4 & NVSSJ040946-040003 & $0.89(0.03)$ & $2.38(0.04)$ & 46.4 & $\mathrm{~N}, \mathrm{M}$ & ? & $1 !, 3 !$ \\
\hline 2FGLJ0420.9-3743 & J042025.09-374445.0 & NVSSJ042025-374443 & $0.78(0.04)$ & $2.44(0.10)$ & 20.2 & $\mathrm{~N}, \mathrm{~S}$ & ? & $3 !$ \\
\hline 2FGLJ0600.9+3839 & J060102.86+383829.2 & NVSSJ060102+383828 & $0.97(0.04)$ & $2.47(0.08)$ & 38.3 & $\mathrm{~N}$ & ? & $2 !, 3 !$ \\
\hline 2FGLJ0644.6+6034 & J064435.72+603851.2 & NVSSJ064435+603849 & $0.64(0.05)$ & $1.97(0.18)$ & 24.6 & $\mathrm{~N}$ & ? & $1,2 !, 3$ \\
\hline 2FGLJ0658.4+0633 & J065845.02+063711.5 & NVSSJ065844+063711 & $0.68(0.04)$ & $1.98(0.15)$ & 27.4 & $\mathrm{~N}$ & $?$ & 3 \\
\hline 2FGLJ0723.9+2901 & J072354.83+285929.9 & NVSSJ072354+285930 & $1.15(0.05)$ & $2.90(0.05)$ & 81.0 & $\mathrm{~N}, \mathrm{~F}$ & ? & $1 !, 2 !, 3 !$ \\
\hline 2FGLJ0746.0-0222 & J074627.03-022549.3 & NVSSJ074627-022549 & $0.68(0.04)$ & $2.11(0.07)$ & 31.3 & $\mathrm{~N}, \mathrm{M}$ & ? & $1 !, 3 !$ \\
\hline 2FGLJ0928.8-3530 & J092849.83-352948.9 & NVSSJ092849-352947 & $0.97(0.04)$ & $2.63(0.05)$ & 57.8 & $\mathrm{~N}, \mathrm{~S}, \mathrm{M}$ & ? & - \\
\hline 2FGLJ1016.1+5600 & $\mathrm{J} 101544.44+555100.7$ & NVSSJ $101544+555100$ & $1.05(0.06)$ & $3.08(0.09)$ & 48.0 & $\mathrm{~N}, \mathrm{~F}, \mathrm{~s}$ & ? & $1 !, 2 !$ \\
\hline 2FGLJ1115.0-0701 & J111511.74-070239.9 & NVSSJ111511-070238 & $0.86(0.06)$ & $2.65(0.15)$ & 17.2 & $\mathrm{~N}$ & $?$ & 3 \\
\hline 2FGLJ1123.3-2527 & $\mathrm{J} 112325.38-252857.0$ & NVSSJ112325-252858 & $0.84(0.03)$ & $2.49(0.03)$ & 30.0 & $\mathrm{~N}, \mathrm{M}, 6, \mathrm{QSR}$ & 0.146 & - \\
\hline 2 FGLJ1129.5+3758 & $\mathrm{J} 112903.25+375657.4$ & NVSSJ112903+375655 & $0.92(0.07)$ & $2.41(0.14)$ & 42.3 & $\mathrm{~N}, \mathrm{~F}, \mathrm{M}, \mathrm{s}, \mathrm{BL} ?$ & ? & 3 \\
\hline 2 FGLJ1223.3+7954 & $\mathrm{J} 122358.17+795327.8$ & NVSSJ122358+795329 & $0.48(0.04)$ & $1.92(0.11)$ & 9.6 & $\mathrm{~N}, \mathrm{M}$ & ? & $2 !, 3$ \\
\hline 2FGLJ1254.2-2203 & J125422.47-220413.6 & NVSSJ125422-220413 & $0.67(0.04)$ & $2.33(0.08)$ & 11.4 & $\mathrm{~N}, \mathrm{M}, \mathrm{v}$ & ? & $1 !, 3$ ! \\
\hline 2FGLJ1259.8-3749 & $\mathrm{J} 125949.80-374858.1$ & NVSSJ125949-374856 & $0.71(0.04)$ & $2.11(0.08)$ & 36.8 & $\mathrm{~N}, \mathrm{~S}, \mathrm{M}, \mathrm{v}$ & ? & $1 !, 3 !$ \\
\hline 2FGLJ1340.5-0412 & $\mathrm{J} 134042.02-041006.8$ & NVSSJ 134042-041006 & $0.71(0.04)$ & $2.12(0.08)$ & 36.6 & $\mathrm{~N}, \mathrm{M}, \mathrm{v}$ & ? & $1 !$ \\
\hline 2FGLJ1347.0-2956 & J134706.89-295842.3 & NVSSJ134706-295840 & $0.79(0.03)$ & $2.11(0.06)$ & 39.8 & $\mathrm{~N}, \mathrm{~S}, \mathrm{M}, \mathrm{v}$ & ? & $1 !, 3 !$ \\
\hline 2FGLJ1513.5-2546 & J151303.66-253925.9 & NVSSJ151303-253924 & $1.01(0.15)$ & $2.65(0.46)$ & 65.9 & $\mathrm{~N}$ & ? & 3 \\
\hline 2 FGLJ1517.2+3645 & $\mathrm{J} 151649.26+365022.9$ & NVSSJ151649+365023 & $0.95(0.03)$ & $2.63(0.04)$ & 54.5 & $\mathrm{~N}, \mathrm{~F}, \mathrm{~s}, \mathrm{v}$ & ? & $1 !, 2,3$ \\
\hline 2 FGLJ1548.3+1453 & $\mathrm{J} 154824.39+145702.8$ & NVSSJ $154824+145702$ & $0.74(0.05)$ & $2.11(0.19)$ & 39.6 & $\mathrm{~N}, \mathrm{~F}, \mathrm{M}, \mathrm{s}$ & ? & - \\
\hline 2 FGLJ $1647.0+4351$ & $\mathrm{~J} 164619.95+435631.0$ & NVSSJ $164619+435631$ & $0.77(0.04)$ & $2.09(0.09)$ & 38.1 & $\mathrm{~N}, \mathrm{~F}, \mathrm{~s}, \mathrm{X}$ & ? & $1 !$ \\
\hline 2 FGLJ1704.3+1235 & $\mathrm{J} 170409.59+123421.7$ & NVSSJ $170409+123421$ & $0.74(0.04)$ & $2.05(0.07)$ & 35.4 & $\mathrm{~N}, \mathrm{M}$ & ? & 3 \\
\hline 2FGLJ1704.6-0529 & J170433.84-052840.6 & NVSSJ $170433-052839$ & $0.78(0.05)$ & $2.14(0.16)$ & 43.0 & $\mathrm{~N}, \mathrm{M}, \mathrm{v}$ & ? & 3 \\
\hline 2FGLJ2004.6+7004 & $\mathrm{J} 200506.02+700439.3$ & NVSSJ200506+700440 & $0.77(0.03)$ & $2.20(0.05)$ & 45.7 & $\mathrm{~N}, \mathrm{v}$ & ? & $1 !, 3$ \\
\hline 2FGLJ2021.5+0632 & $\mathrm{J} 202155.45+062913.7$ & NVSSJ202155+062914 & $0.82(0.03)$ & $2.12(0.05)$ & 35.3 & $\mathrm{~N}, \mathrm{M}$ & ? & $1 !, 3 !$ \\
\hline 2 FGLJ2115.4+1213 & $\mathrm{J} 211522.00+121802.8$ & NVSSJ211522+121802 & $0.78(0.05)$ & $2.23(0.18)$ & 46.2 & $\mathrm{~N}, \mathrm{M}$ & ? & $3 !$ \\
\hline 2FGLJ2132.5+2605 & $\mathrm{J} 213253.05+261143.8$ & NVSSJ $213252+261143$ & $1.20(0.05)$ & $2.78(0.09)$ & 25.9 & $\mathrm{~N}$ & $?$ & 3 \\
\hline 2FGLJ2133.9+6645 & $\mathrm{J} 213349.21+664704.3$ & NVSSJ213349+664706 & $0.80(0.04)$ & $2.28(0.06)$ & 49.0 & $\mathrm{~N}, \mathrm{v}$ & ? & $1 !, 2,3$ \\
\hline 2FGLJ2134.6-2130 & J213430.18-213032.6 & NVSSJ213430-213032 & $0.78(0.04)$ & $2.27(0.08)$ & 44.3 & $\mathrm{~N}, \mathrm{M}$ & ? & $1 !, 3$ \\
\hline 2FGLJ2228.6-1633 & $\mathrm{J} 222830.19-163642.8$ & NVSSJ 222830-163643 & $0.74(0.04)$ & $2.23(0.12)$ & 37.9 & $\mathrm{~N}, \mathrm{M}$ & $?$ & $3 !$ \\
\hline 2FGLJ2246.3+1549 & $\mathrm{J} 224604.98+154435.3$ & NVSSJ 224604+154437 & $0.61(0.05)$ & $2.17(0.14)$ & 16.0 & $\mathrm{~N}, \mathrm{M}$ & ? & $3 !$ \\
\hline 2FGLJ2358.4-1811 & J235836.83-180717.3 & NVSSJ 235836-180718 & $0.86(0.04)$ & $2.21(0.10)$ & 43.2 & $\mathrm{~N}, \mathrm{M}, 6, \mathrm{X}, \mathrm{BL}$ & $0.058 ?$ & 1 \\
\hline \multicolumn{9}{|c|}{ SOUTHERN UGS SAMPLE } \\
\hline 2FGLJ0116.6-6153 & J011619.59-615343.5 & SUMSSJ011619-615343 & $0.85(0.04)$ & $2.34(0.06)$ & 49.9 & $\mathrm{~S}, \mathrm{M}$ & ? & $1 !, 3 !$ \\
\hline 2FGLJ0133.4-4408 & J013306.35-441421.3 & SUMSSJ013306-441422 & $0.83(0.03)$ & $2.25(0.05)$ & 51.0 & $\mathrm{~S}, \mathrm{M}$ & ? & $1 !, 3 !$ \\
\hline 2FGLJ0143.6-5844 & J014347.39-584551.3 & SUMSSJ014347-584550 & $0.69(0.03)$ & $1.93(0.06)$ & 23.0 & $\mathrm{~S}, \mathrm{M}$ & ? & $1 !, 3$ \\
\hline 2FGLJ0316.1-6434 & J031614.31-643731.4 & SUMSSJ031614-643732 & $0.74(0.03)$ & $2.10(0.06)$ & 38.9 & $\mathrm{~S}, \mathrm{M}$ & ? & $1 !, 3$ \\
\hline 2FGLJ0416.0-4355 & J041605.81-435514.6 & SUMSSJ041605-435516 & $1.11(0.03)$ & $2.90(0.04)$ & 97.2 & $\mathrm{~S}, \mathrm{M}$ & ? & $1 !$ \\
\hline 2FGLJ0420.9-3743 & J042025.09-374445.0 & MRSS303-096250 & $0.78(0.04)$ & $2.44(0.10)$ & 20.2 & $\mathrm{~N}, \mathrm{~S}$ & ? & $3 !$ \\
\hline 2FGLJ0555.9-4348 & J055618.74-435146.1 & SUMSSJ055618-435146 & $0.91(0.03)$ & $2.50(0.04)$ & 43.9 & $\mathrm{~S}, \mathrm{M}$ & ? & $1 !$ \\
\hline 2FGLJ0928.8-3530 & J092849.83-352948.9 & SUMSSJ092849-352947 & $0.97(0.04)$ & $2.63(0.05)$ & 57.8 & $\mathrm{~N}, \mathrm{~S}, \mathrm{M}$ & $?$ & - \\
\hline 2FGLJ1032.9-8401 & J103015.35-840308.7 & SUMSSJ103014-840307 & $0.99(0.04)$ & $2.63(0.05)$ & 62.1 & $\mathrm{~S}, \mathrm{v}$ & $?$ & $1 !$ \\
\hline 2FGLJ1259.8-3749 & $\mathrm{J} 125949.80-374858.1$ & SUMSSJ125949-374856 & $0.71(0.04)$ & $2.11(0.08)$ & 36.8 & $\mathrm{~N}, \mathrm{~S}, \mathrm{M}, \mathrm{v}$ & ? & $1 !, 3 !$ \\
\hline 2FGLJ1328.5-4728 & $\mathrm{J} 132840.61-472749.2$ & SUMSSJ132840-472748 & $0.63(0.04)$ & $2.08(0.08)$ & 24.4 & $\mathrm{~S}, \mathrm{M}, \mathrm{v}$ & ? & $3 !$ \\
\hline 2FGLJ2042.8-7317 & J204201.92-731913.5 & SUMSSJ204201-731911 & $0.65(0.05)$ & $1.81(0.16)$ & 12.1 & $\mathrm{~S}, \mathrm{M}$ & ? & - \\
\hline 2FGLJ2131.0-5417 & $\mathrm{J} 213208.28-542036.4$ & SUMSSJ213208-542037 & $1.25(0.09)$ & $2.92(0.19)$ & 29.0 & $\mathrm{~s}$ & ? & - \\
\hline 2FGLJ2213.7-4754 & $\mathrm{J} 221330.33-475425.0$ & SUMSSJ 221330-475426 & $0.90(0.04)$ & $2.23(0.10)$ & 33.4 & $\mathrm{~S}, \mathrm{M}$ & ? & - \\
\hline
\end{tabular}

Col. (1) 2FGL name.

Col. (2) WISE name.

Col. (3) Radio name.

Cols. (4,5) IR colors from WISE. Values in parentheses are $1 \sigma$ uncertainties.

Col. (6) Notes: $\mathrm{N}=$ NVSS, $\mathrm{F}=$ FIRST, $\mathrm{M}=2 \mathrm{MASS}$, $\mathrm{s}=\mathrm{SDSS} \mathrm{dr} 9,6=6 \mathrm{dFG}$; $\mathrm{X}=\mathrm{ROSAT}$; $\mathrm{QSO}=$ quasar, $\mathrm{BL}=\mathrm{BL}$ Lac; $\mathrm{v}=$ variable in WISE bands (var_flag $>5$ in at least one band, see Cutri et al. 2012 for additional details); rv = variable in the radio bands at $1.4 \mathrm{GHz}$.

Col. (7) Estimate level of confide
Col. (8) Redshift: ? = unknown.

Col. (9) Redsults : $=$ the in Paggi et al. (2013). Exclamation mark (!) indicates that the $\gamma$-ray blazar candidate is the same IR source found in the previous investigation.

and those presented in Massaro et al. (2013b) based on the WENSS radio analysis was not possible because the footprints of the surveys used did not overlap. We also verified that the selected $\gamma$-ray blazar candidates having a SDSS counterpart exhibit optical color consistent with those of BL Lacs (i.e., $u-r<1.4$, see Massaro et al. 2012, for more details). We found that with the only exception of NVSSJ154824+145702 all of them have the same optical properties of the BZB population.

Within the whole sample of UGSs analyzed, there are
25 sources that were also unidentified in the 1FGL (? ) and were analyzed on the basis of two different statistical approaches: the Classification Tree and the Logistic regression analyses (see Ackermann et al. 2012, and references therein). By comparing the results of our association method with those in Ackermann et al. (2012), we found that 19 out of 25 UGSs with a $\gamma$-ray blazar candidate recognized according to our method are also classified as AGNs. All of them with a probability higher than $60 \%$ with 14 higher than $80 \%$. The remaining three sources were classified as pulsar candidates but with a 
TABLE 3

Unidentified Gamma-RAY SOURCES IN the SDA SAMPLE.

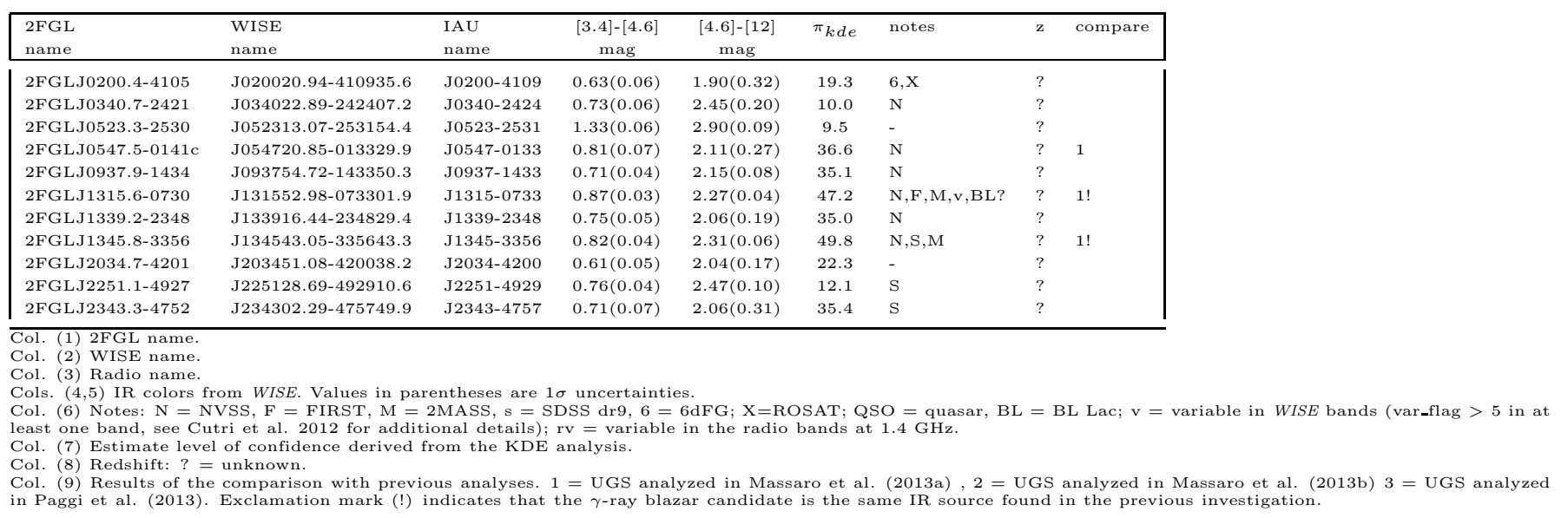

very low probability (i.e. $\leq 60 \%$ ) Consequently, our results are in good agreement with the classification suggested previously by Ackermann et al. (2012) and thus consistent with the $\gamma$-ray AGN nature.

Finally, we remark that several $\gamma$-ray pulsars have been identified after the release of the 2FGL, where they are listed as UGSs. However, we did not exclude these UGSs from our sample to test if, as expected, we did not find any blazar-like counterpart associable to them. Thus, in agreement with our expectations, all the UGSs for which we found a $\gamma$-ray blazar candidates do not have any pulsars associated according to the Public List of LAT-Detected Gamma-Ray Pulsars 9 .

\section{SUMMARY AND CONCLUSIONS}

In this paper we presented an non-parametric method to search for $\gamma$-ray blazar candiates within two samples of UGSs. First we identify all the radio sources in the two major surveys (i.e., NVSS and SUMSS Condon et al. 1998; Mauch et al. 2003, respectively) that lie within the positional uncertainty region at $95 \%$ level of confidence, then we investigate the IR colors of their WISE counterparts to recognize those with similar spectral properties in the simple [3.4][4.6]-[12] color-color plot. With respect to our previous WISE selection of $\gamma$-ray blazar candidates (e.g., Massaro et al. 2012a; D'Abrusco et al. 2013) the criteria adopted in the present analysis are less conservative, since the detection of the WISE counterpart at $22 \mu \mathrm{m}$ is not required. A small fraction $(\sim 8 \%)$ of the Fermi blazar are in fact not detected at $22 \mu \mathrm{m}$. Thus, to compare the IR colors of the Fermi blazars with those of the radio sources selected, we adopted a KDE technique as already presented in Massaro et al. (2011a), Massaro et al. (2012a) and more recently in Paggi et al. (2013). Our new approach, being less restrictive than those adopted in our previous associations, permits to search for faint $\gamma$-ray blazar candidates that were not previously selected because too faint at $22 \mu \mathrm{m}$. By relaxing the requirement on the detection at $22 \mu \mathrm{m}$ and thus on the [12]-[22] color, this method would select candidate blazars at the cost of a larger contamination, mitigated by the requirement on the presence of a radio counterpart.
We found 41 and 14 radio sources with IR similar to those of the Fermi blazars within the NU and the SU samples, respectively. In addition, we investigated the sample of radio sources discovered with recent deep ATCA observations performed to search for radio counterparts of the UGS in the southern hemisphere. Among 416 radio objects listed in Petrov et al. (2013) only 11 sources have an IR counterpart consistent with the $\gamma$-ray blazars. The total number of $\gamma$-ray blazar candidates is 66 all listed in Table 2 and Table 3 , without no multiple candidates within the positional uncertainty regions of the UGSs analyzed. We estimate a probability of spurious association for the $\gamma$-ray blazar candidates selected according to our method of the order of $4 \%$ and $3 \%$ for the NU and SU samples, respectively.

It is worth noting that the large majority of our candidates show IR colors more consistent with the region occupied by the BZBs in the [3.4]-[4.6]-[12] $\mu \mathrm{m}$ color-color diagram rather than that of BZQs. Thus they could be potential faint and so distant BZBs that were not previously selected with different methods because lacking of the IR flux at $22 \mu \mathrm{m}$. More detailed investigations based on ground-based, optical and near IR, spectroscopic follow up observations will be planned for the selected $\gamma$-ray blazar candidates to confirm their nature and to obtain their redshifts.

We thank the anonymous referee for useful comments on the probability of spurious associations that improved our paper. The work is supported by the NASA grants NNX12AO97G. R. D'Abrusco gratefully acknowledges the financial support of the US Virtual Astronomical Observatory, which is sponsored by the National Science Foundation and the National Aeronautics and Space Administration. The work by G. Tosti is supported by the ASI/INAF contract I/005/12/0. Howard A. Smith acknowledges partial support from NASA-JPLRSA contract 717437. TOPCATH (Taylor 2005) for the preparation and manipulation of the tabular data and the images. The WENSS project was a collaboration between the Netherlands Foundation for Research in Astronomy and the Leiden Observatory. We acknowledge

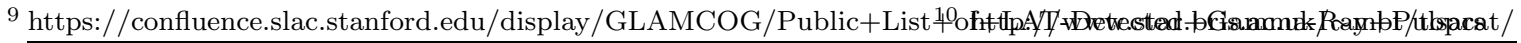


TABLE 4

OPticAl MAGNitUdes FOR THE WISE COUNTERPARTS.

\begin{tabular}{|c|c|c|c|c|c|c|}
\hline $\begin{array}{l}\text { WISE } \\
\text { name }\end{array}$ & $\begin{array}{c}\mathrm{B} 1 \\
\mathrm{mag}\end{array}$ & $\begin{array}{c}\mathrm{R} 1 \\
\mathrm{mag}\end{array}$ & $\begin{array}{c}\mathrm{B} 2 \\
\mathrm{mag}\end{array}$ & $\begin{array}{c}\mathrm{R} 2 \\
\mathrm{mag}\end{array}$ & $\begin{array}{c}\mathrm{I} \\
\mathrm{mag}\end{array}$ & $\begin{array}{c}\theta \\
\operatorname{arcsec}\end{array}$ \\
\hline J003119.70+072453.6 & 19.03 & 18.17 & 19.84 & 18.63 & 18.67 & 0.14 \\
\hline J003908.14+433014.6 & 19.9 & 19.61 & 21.42 & 20.77 & & 0.14 \\
\hline J010345.73+132345.4 & 17.98 & 17.73 & 18.69 & 17.38 & 17.24 & 0.07 \\
\hline J011619.59-615343.5 & & 17.72 & 18.22 & 17.78 & 17.91 & 0.27 \\
\hline J013306.35-441421.3 & & 18.38 & 19.7 & 18.12 & 18.76 & 0.26 \\
\hline J014347.39-584551.3 & & 16.7 & 18.48 & 16.64 & 17.04 & 0.04 \\
\hline J015248.80+855703.6 & 20.57 & 18.84 & 19.63 & 18.71 & 17.82 & 0.38 \\
\hline J020020.94-410935.6 & & 19.84 & 21.1 & 18.79 & 18.75 & 0.6 \\
\hline $\mathrm{J} 022744.35+224834.3$ & & & 20.82 & 20.22 & 19.28 & 0.35 \\
\hline J031240.54+201142.8 & & 19.34 & 21.22 & 19.42 & 19.07 & 2.63 \\
\hline J031614.31-643731.4 & & 16.59 & 18.19 & 16.57 & 16.82 & 0.22 \\
\hline $\mathrm{J} 033153.90+630814.1$ & & & 20.66 & 19.92 & 18.35 & 0.35 \\
\hline J034022.89-242407.2 & & 19.56 & 20.07 & & & 0.21 \\
\hline J035309.54+565430.8 & 20.09 & 19.24 & 20.43 & 18.76 & 18.53 & 0.55 \\
\hline J040946.57-040003.4 & 19.45 & 19.18 & 17.53 & 16.98 & 16.86 & 0.07 \\
\hline J041605.81-435514.6 & & 18.49 & 18.7 & 18.17 & 18.0 & 0.18 \\
\hline J042025.09-374445.0 & & 20.44 & 20.73 & 19.71 & 18.17 & 0.38 \\
\hline J052313.07-253154.4 & & 19.2 & 20.83 & 20.07 & 18.95 & 0.17 \\
\hline J055618.74-435146.1 & & 19.23 & 18.88 & 19.08 & 18.08 & 0.31 \\
\hline J060102.86+383829.2 & & 19.11 & & 19.84 & 18.48 & 0.04 \\
\hline J064435.72+603851.2 & 20.01 & 19.58 & 20.7 & 18.75 & 18.37 & 0.3 \\
\hline J065845.02+063711.5 & 20.25 & & & 19.12 & 18.3 & 0.39 \\
\hline J072354.83+285929.9 & 19.78 & 19.05 & 19.97 & 18.72 & & 0.19 \\
\hline J074627.03-022549.3 & 19.03 & & 18.59 & 18.43 & 16.53 & 0.31 \\
\hline J092849.83-352948.9 & & 18.56 & 19.64 & 18.07 & 18.23 & 0.23 \\
\hline J093754.72-143350.3 & 18.82 & 17.92 & 18.64 & 17.73 & 17.56 & 0.1 \\
\hline $\mathrm{J} 101544.44+555100.7$ & 19.69 & 19.42 & 20.61 & 19.35 & & 0.37 \\
\hline J103015.35-840308.7 & & 19.36 & 19.26 & 18.84 & 18.03 & 0.15 \\
\hline J111511.74-070239.9 & & 19.86 & 20.68 & 19.05 & 18.66 & 0.14 \\
\hline J112325.38-252857.0 & 16.9 & 15.76 & 15.87 & 15.56 & 15.51 & 0.19 \\
\hline $\mathrm{J} 112903.25+375657.4$ & 19.9 & 19.23 & 19.35 & 19.48 & 18.58 & 0.65 \\
\hline $\mathrm{J} 122358.17+795327.8$ & & 17.6 & 20.18 & 18.46 & 17.63 & 1.04 \\
\hline J125422.47-220413.6 & & 19.88 & 18.67 & 19.11 & 18.22 & 0.41 \\
\hline J125949.80-374858.1 & & 17.44 & 18.07 & 16.78 & 17.35 & 0.17 \\
\hline $\mathrm{J} 131552.98-073301.9$ & 19.78 & 18.68 & 18.75 & 17.75 & 17.56 & 0.16 \\
\hline $\mathrm{J} 132840.61-472749.2$ & & 17.75 & 18.23 & 16.8 & & 0.98 \\
\hline $\mathrm{J} 133916.44-234829.4$ & 20.3 & 19.3 & 20.43 & 19.79 & 18.5 & 0.31 \\
\hline $\mathrm{J} 134042.02-041006.8$ & 18.21 & 17.21 & 17.59 & 16.46 & 17.08 & 0.19 \\
\hline J134543.05-335643.3 & & 17.98 & 19.58 & 18.65 & 18.12 & 0.38 \\
\hline J134706.89-295842.3 & 17.85 & 17.09 & 18.8 & 17.14 & 17.09 & 0.41 \\
\hline J151303.66-253925.9 & 19.92 & 18.96 & 19.77 & 20.35 & & 0.5 \\
\hline $\mathrm{J} 151649.26+365022.9$ & 20.9 & & 21.49 & 20.07 & 19.16 & 1.58 \\
\hline $\mathrm{J} 154824.39+145702.8$ & 20.51 & 18.29 & 19.86 & 17.74 & 17.45 & 0.41 \\
\hline $\mathrm{J} 164619.95+435631.0$ & 20.43 & 19.73 & 20.42 & 19.67 & & 0.34 \\
\hline $\mathrm{J} 170409.59+123421.7$ & 19.86 & 18.04 & 18.62 & 17.63 & 17.46 & 0.47 \\
\hline J170433.84-052840.6 & 19.62 & 18.97 & 18.42 & 17.28 & 17.98 & 0.45 \\
\hline $\mathrm{J} 200506.02+700439.3$ & 20.73 & 19.25 & 19.24 & 18.65 & & 0.45 \\
\hline $\mathrm{J} 202155.45+062913.7$ & 17.27 & 16.13 & 17.01 & 16.67 & 16.03 & 0.43 \\
\hline $\mathrm{J} 203451.08-420038.2$ & & 18.97 & 19.34 & 18.87 & 18.27 & 0.44 \\
\hline J204201.92-731913.5 & & 17.46 & 17.9 & 18.36 & 18.04 & 0.29 \\
\hline $\mathrm{J} 211522.00+121802.8$ & 18.15 & 18.15 & 17.68 & 17.31 & 17.58 & 0.16 \\
\hline $\mathrm{J} 213253.05+261143.8$ & 20.04 & 19.29 & 19.14 & 19.62 & 18.44 & 0.07 \\
\hline J213430.18-213032.6 & 19.77 & 18.65 & 18.96 & 16.8 & 17.7 & 0.09 \\
\hline $\mathrm{J} 213349.21+664704.3$ & & & & 19.37 & 18.8 & 0.45 \\
\hline $\mathrm{J} 221330.33-475425.0$ & & 18.12 & 18.6 & 18.34 & 18.33 & 0.05 \\
\hline J222830.19-163642.8 & 18.57 & 19.34 & 19.95 & 19.04 & 17.91 & 0.29 \\
\hline $\mathrm{J} 224604.98+154435.3$ & 19.14 & 18.27 & 19.57 & 18.53 & 17.65 & 0.13 \\
\hline J225128.69-492910.6 & & 18.8 & 19.21 & 18.45 & 18.03 & 0.42 \\
\hline $\mathrm{J} 234302.29-475749.9$ & & 19.84 & 18.92 & 21.3 & 18.32 & 0.29 \\
\hline J235836.83-180717.3 & 19.14 & 18.45 & 18.28 & 17.22 & 17.53 & 0.3 \\
\hline
\end{tabular}

the WENSS team consisted of Ger de Bruyn, Yuan Tang, Roeland Rengelink, George Miley, Huub Rottgering, Malcolm Bremer, Martin Bremer, Wim Brouw, Ernst Raimond and David Fullagar for the extensive work aimed at producing the WENSS catalog. Part of this work is based on archival data, software or on-line services provided by the ASI Science Data Center. This research has made use of data obtained from the High Energy Astrophysics Science Archive Research Center (HEASARC) provided by NASA's Goddard Space Flight Center; the SIMBAD database operated at CDS, Strasbourg, France; the NASA/IPAC Extragalactic Database (NED) operated by the Jet Propulsion Laboratory, California Institute of Technology, under contract with the National Aeronautics and Space Administration. Part of this work is based on the NVSS (NRAO VLA Sky Survey); The National Radio Astronomy Observatory is operated by Associated Universities, Inc., under contract with the National Science Foundation. This publication makes use of data products from the Two Micron All Sky Survey, which is a joint project of the University of Massachusetts and the Infrared Processing and Analysis Center/California Institute of Technology, funded by the National Aeronautics and Space Administration and the National Science Foundation. This publication makes use of data products from the Wide-field Infrared Survey Explorer, which is a joint project of the University of California, Los Angeles, and the Jet Propulsion Laboratory/California Institute of Technology, funded by the National Aeronautics and Space Administration.

\section{REFERENCES}

Abdo, A. A. et al. 2010a ApJS 188405

Abdo, A. A. et al. 2010b ApJ, 720, 435

Ackermann, M. et al. 2011b ApJ, 743, 171

Ackermann, M. et al. 2011a ApJ, 741, 30

Ackermann, M. et al. 2012 ApJ, 753, 83

Adelman-McCarthy, J., Agueros, M.A., Allam, S.S., et al. 2008, ApJS, 175, 297

Becker, R. H., White, R. L., Helfand, D. J.1995 ApJ, 450, 559

Condon, J. J., Cotton, W. D., Greisen, E. W., Yin, Q. F., Perley, R. A., Taylor, G. B., \& Broderick, J. J. 1998, AJ, 115, 1693 Cutri et al. 2012 wise.rept, $1 \mathrm{C}$

D'Abrusco, R., Longo, G., Walton, N. A. 2009 MNRAS , 396, 223

D'Abrusco, R., Massaro, F., Ajello, M., Grindlay, J. E., Smith,

Howard A. \& Tosti, G. 2012 ApJ, 748, 68

D'Abrusco, R., Massaro, F., Paggi, A., Masetti, N., Giroletti, M., Tosti, G., Smith, Howard, A. 2013 ApJS, 206, 12
Draine B. T. 2003 ARA\&A, 41, 241

Ghirlanda, G., Ghisellini, G., Tavecchio, F., Foschini, L. 2010 MNRAS, 407, 791

Hartman, R.C. et al., 1999 ApJS, 123, 79

Kovalev, Y. Y. 2009 ApJ, 707L, 56

Kovalev, Y. Y. et al. 2009 ApJ, 696L, 17

Jones, H. D. et al. 2004 MNRAS, 355, 747

Jones, H. D. et al. 2009 MNRAS, 399, 683

Laurino, O. \& D'Abrusco 2011 MNRAS in press

Mahony, E. K., Sadler, E. M., Murphy, T., Ekers, R. D. Edwards, P. G., Massardi, M. 2010 ApJ, 718, 587

Massaro, E., Giommi, P., Leto, C., Marchegiani, P., Maselli, A., Perri, M., Piranomonte, S., Sclavi, S. 2009 A\&A, 495, 691

Massaro, E., Giommi, P., Leto, C., Marchegiani, P., Maselli, A., Perri, M., Piranomonte, S., Sclavi, S. 2010

http://arxiv.org/abs/1006.0922 
Massaro, F., D'Abrusco, R., Ajello, M., Grindlay, J. E. \& Smith, H. A. 2011a ApJ, 740L, 48

Massaro, E., Giommi, P., Leto, C., Marchegiani, P., Maselli, A., Perri, M., Piranomonte, S., 2011b "Multifrequency Catalogue of Blazars (3rd Edition)", ARACNE Editrice, Rome, Italy

Massaro, F., D'Abrusco, R., Tosti, G., Ajello, M., Gasparrini, D., Grindlay, J. E. \& Smith, Howard A. 2012a ApJ, 750, 138

Massaro, F., D'Abrusco, R., Tosti, G., Ajello, M., Paggi, A., Gasparrini, D. 2012b ApJ, 752, 61

Massaro, E., Nesci, R., Piranomonte, S. 2012 MNRAS ,422, 2322

Massaro, F. et al. 2013a ApJS, 206, 13

Massaro, F. et al. 2013b ApJS, 207, 4

Mauch, T., Murphy, T., Buttery, H. J., Curran, J., Hunstead, R. W., Piestrzynski, B., Robertson, J. G., Sadler, E. M. 2003 MNRAS, 342, 1117

Mirabal, N. 2009 [arxiv.org/abs/0908.1389v2]

Mirabal, N. 2009 ApJ, 701, 129

Mirabal, N, D. \& Pardo, S. 2010 A\&A submitted, [arxiv.org/abs/1007.2644v2]

Monet, D. G. et al. 2003 AJ, 125, 984
Mukherjee, R. et al., 1997 ApJ, 490, 116

Nolan et al. 2012 ApJS, 199, 31

Petrov, L., Mahony, E. K., Edwards, P. G., Sadler, E. M.,

Schinzel, F. K., McConnell, D. 2013 MNRAS, 432, 1294

Paggi, A., Massaro, F., D'Abrusco, R. et al. 2013 ApJS submitted

Paris, I. et al. 2012 A\&A, 548A, 66

Rengelink, R. et al. 1997, A\&A Suppl. 124, 259

Richards, G. T. et al., 2004 ApJS, 155, 257

Skrutskie, M. F. et al. 2006, AJ, 131, 1163

Takeuchi Y. et al., 2013, ApJS submitted

Taylor M. B., 2005, ASPC, 347, 29

Thompson, D. J. 2008 RPPh, 71, 11, 116901

Ungruhe, R., Seitter, W. C., Duerbeck, H. W. 2003 JAD, 9, 1

Voges, W. et al. 1999 A\&A, 349, 389

White, R. L., Becker, R. H. Helfand, D. J., Gregg, M. D. et al. 1997 ApJ, 475, 479

Wright, E. L., et al. 2010 AJ, 140, 1868

Zechlin, H.-S., Fernandes, M. V., Elsasser, D., Horns, D. 2012 A\&A, 538A, 93 